\title{
Display of Vector Fields Using a Reaction-Diffusion Model
}

\author{
Allen R. Sanderson, Chris R. Johnson, and Robert M. Kirby* \\ Scientific Computing and Imaging Institute, University of Utah
}

\begin{abstract}
Effective visualization of vector fields relies on the ability to control the size and density of the underlying mapping to visual cues used to represent the field. In this paper we introduce the use of a reaction-diffusion model, already well known for its ability to form irregular spatio-temporal patters, to control the size, density, and placement of the vector field representation. We demonstrate that it is possible to encode vector field information (orientation and magnitude) into the parameters governing a reaction-diffusion model to form a spot pattern with the correct orientation, size, and density, creating an effective visualization. To encode direction we texture the spots using a light to dark fading texture. We also show that it is possible to use the reaction-diffusion model to visualize an additional scalar value, such as the uncertainty in the orientation of the vector field.

An additional benefit of the reaction-diffusion visualization technique arises from its automatic density distribution. This benefit suggests using the technique to augment other vector visualization techniques. We demonstrate this utility by augmenting a LIC visualization with a reaction-diffusion visualization. Finally, the reaction-diffusion visualization method provides a technique that can be used for streamline and glyph placement.
\end{abstract}

Keywords: Vector Field Visualization, Flow Visualization, Reaction-Diffusion, Vector Fields

\section{INTRODUCTION}

Visualizing vector fields is important for many computational applications, including fluid dynamics, wind and water currents in climate modeling, bioelectric fields in neuroscience, and magnetic fields in nuclear fusion. To meet the needs arising from this diverse set of applications, many different techniques for visualizing vector fields have been developed $[1,3,7,8,19,22,31,33,30]$. Each technique has strengths and weaknesses in its ability to represent the magnitude, orientation, direction, uncertainty and topological structures of the associated vector field.

For instance, the simplest method for displaying a vector field is to place glyphs representing the vector direction and magnitude at regular intervals. However, because of scaling differences, overlap between the glyphs can occur. This can produce visual clutter and occlusion [25]. The problem is further compounded when data are displayed in three dimensions. Displaying normalized vector values can reduce the clutter but at a loss of information. Even when the visual clutter can be overcome, displaying vector fields using regular intervals may not be appropriate. This is because the grid spacing may not correspond to the underlying vector field.

More complicated techniques, such as streamlines, can provide powerful visual cues [10]. However, enough streamlines must be placed to provide cues without causing visual clutter. Streamlines

*e-mail: \{allen,crj,kirby\}@sci.utah.edu

IEEE Visualization 2004

October 10-15, Austin, Texas, USA

0-7803-8788-0/04/\$20.00 @2004 IEEE may be placed selectively to reduce the clutter, but such selective placement may cause critical areas to be missed [28].

With the exception of a glyph-based method, no technique is singularly able to visualize uncertainty in vector fields. In [17] Pang et al. demonstrated several different glyphs for characterizing uncertainty in vector fields. However, as a glyph based method it can also succumbs to clutter and occlusion.

Given the various shortcomings in many of the current vector field visualization techniques, the main goal of this work was to develop an automated method that uses the vector magnitude, orientation, direction, and uncertainty to control the shape, size, orientation, direction, and density of the objects used to represent the vector field. At the same time, we wanted a method that would be mesh independent and produce a visualization that would be natural and pleasing to the eye. To achieve these goals, we have explored the use of a reaction-diffusion model for vector field visualization.

\section{Background And Previous Work}

Because of its importance to scientific computing applications, creating effective techniques for visualizing vector fields is an active area of research. Given the large number of techniques that have been developed, it is not practical to review each technique; a very complete review can be found in [18]. Instead, we focus on three related areas for visualizing vector fields: the use of random patterns, selective placement, and reaction-diffusion.

The use of random patterns for visualizing a vector field has been explored by van Wijk [29], Cabral and Leedom [3], Shen et al. [23], and others using either spot or white noise to form a dense representation of the vector field by integrating an ODE that represents the basis for a streamline. By dense, we mean that there is value for each grid location. Others, such as Preußer, [20] have formulated the problem as a PDE describing nonlinear anisotropic diffusion. With the ODE and PDE formulations the resulting image has a brush-stroke appearance of variable thickness. While this type of image is useful for showing the vector orientation, it fails to provide information about magnitude and direction. These shortcomings have been addressed in various forms by adding directional $[23,30]$ and magnitude $[5,13]$ cues.

More recent work has focused on creating images that are less dense, but still contain useful information about the vector field, e.g. [12, 24, 28]. Turk and Banks [28] explored a method to bundle similar streamlines until an energy function is minimized. Once the function is minimized, the streamlines can be replaced with variable sized curved arrows to show direction and magnitude. Kirby et al. [12], were able to achieve similar results using a random placement of variable sized arrows. Once an arrow is placed, a Poisson distribution disk based on the vector magnitude is used to prevent other arrows from being placed near it. However, because the arrow represents just the value at a single location rather than representing the values in a neighborhood of the sample, it is possible to create the illusion that an area is homogeneous when it is not.

Computer graphics applications have also made use of a reaction-diffusion model to generate texture maps [27, 32]. These types of textures are useful for forming patterns that are natural looking and are typically applied to organic models such as ani- 
mals. Turk explored the use of different reaction models to produce a variety of patterns [27]. At the same time, Witkin and Kass [32] used anisotropic diffusion to form different patterns. These patterns can be classified as either spot or stripe patterns.

Rather than forming the texture and then applying it to a model using a traditional texture mapping, Turk exploited the fact that a reaction-diffusion model can be used on an irregular grid. This allowed him to create textures directly on a tessellated surface, avoiding any warping between model space and parameter space. It is possible to make use of this same property to texture isosurfaces, which is a very common visualization tool. In a similar vein, Chambers and Rockwood [4] employed a reaction-diffusion model to generate a solid texture, which is used on a surface and on a volume. Like Witkin and Kass, Chambers and Rockwood also used an anisotropic diffusion technique to form stripe patterns.

Although the reaction-diffusion model was initially mentioned by Cabral and Leedom as a possible model for visualizing data [3], the first researchers to use the method were Kindlmann et al.[11]. They created a solid texture using a reaction-diffusion model based on tensor values from diffusion-weighted magnetic resonance images. This anisotropy formed elliptical "blobs," which were then volume rendered. Our approach is similar in that we also use a tensor but rather than having tensor data supplied we create the tensor from vector data. This gives several additional degrees of freedom that can be used to encode additional data.

Perhaps the most closely related work is that of Garcke et al. [6] were they use the vector field to define the amount anisotropy in the diffusion. Further, they are able to incorporate a shaped particle to show direction as well as orientation. They use both methods to create visualizations of clustered vector fields.

We now present a more detailed look at reaction-diffusion, and describe how it can be used to visualize vector field data.

\section{REACTION DIFfusion}

In 1952 Turing [26] proposed a reaction-diffusion model for describing the chemical process between two morphogens within a series of cells. Due to the dynamics of the system, the morphogens both react and diffuse which changes their concentration within each cell. With time, the morphogens may form a stable pattern representing the dynamic equilibrium of the system. The pattern formation is not dependent on the initial state of the system; the dynamics of the system drives the concentrations toward an equilibrium state.

Turing described the reaction-diffusion of a two morphogen model as a set of nonlinear partial differential equations:

$$
\begin{aligned}
& \frac{\mathscr{I} a}{\mathbb{I} t}=F(a, b)+d_{a} \nabla^{2} a \\
& \frac{\mathbb{I} b}{\mathbb{I} t}=G(a, b)+d_{b} \nabla^{2} b
\end{aligned}
$$

where $a$ and $b$ are the morphogen concentrations; $F$ and $G$ are the functions controlling the production rate of $a$ and $b$, respectively; $d_{a}$ and $d_{b}$ are the diffusion rates, and $\nabla^{2} a$ and $\nabla^{2} b$ are the Laplacians of $a$ and $b$, respectively. Turing further defined $F$ and $G$ as:

$$
\begin{gathered}
F(a, b)=s(a-a b) \\
G(a, b)=s(a b-b-b)
\end{gathered}
$$

where $a$ and $b$ again are the morphogen concentrations, $a$ and $b$ are the formation and degradation rates of $a$ and $b$ respectively, and $s$ is the reaction rate.

For the state to change, there must be some perturbation in the initial conditions which is a stable solution. This perturbation can arise from a non-uniformity in either the initial concentrations, $a$ and $b$, or the formation and degradation rates, $a$ and $b$. A nonuniform formation and degradation rate can be interpreted as being the natural variation within each cell.

After the system is put into motion, the morphogen concentrations will change until a dynamic equilibrium is reached and thus a stable pattern is formed. Although the pattern is stable, the morphogen concentration in each cell will continue to change. However, the change is statistically very small.

Turing's equations are just one specific instance of reactiondiffusion phenomena. Other similar variations can be found in the literature, including those in [16]. In this paper, we have focused on the use of Turing's model because it provides results that are indicative of reaction-diffusion in general. We note that the techniques provided in this paper can be similarly applied to other reactiondiffusion systems.

\subsection{Mapping the Reaction-Diffusion Kinetics}

In order to use a reaction-diffusion model for visualization, a mapping must be established between the vector field and the reactiondiffusion model. There are three possibilities: a mapping between the vector field and the reaction kinetics, a mapping between the vector field and the diffusion kinetics, or a combination of both. Because of the instabilities associated with a reaction-diffusion model, we have focused on finding a mapping for the vector magnitude to the reaction kinetics and the vector orientation to the diffusion kinetics.

\subsection{Reaction Kinetics}

Within Turing's reaction kinetics there are several free variables. We first mapped the patterns formed as a function of the two reaction values, $a$ and $b$. The patterns formed can be described as being finger print or spot patterns. However, these formed only inside a very narrow band of values for $a$ and $b$. On either side of this band a stable pattern did not occur. For this work we chose a and $b$ to be $16 \pm 1 \%$ and $12 \pm 1 \%$ respectively, which produced spot like patterns.

The other free parameter in the reaction kinetics is the reaction rate $s$. Changing the reaction rate changes the size of the pattern formed. This provides an ideal mapping to a scalar value such as the vector magnitude. Thus, it is possible to create patterns of varying size with the size directly relating to the vector magnitude.

It should be noted that similar results can be obtained by varying the diffusion rates $d_{a}$ and $d_{b}$. As such, it is neither the reaction nor the diffusion rates that changes the size, but rather their relative difference. For simplicity and clarity, we vary only the reaction rate for each cell.

\subsection{Diffusion Kinetics}

The diffusion kinetics as written in Equations (1) and (2) have one free parameter per equation, the diffusion rates $d_{a}$ and $d_{b}$. As previously noted, changing the diffusion rate changes the size of the pattern formed, and for our purposes it is fixed. However, this is not the only free parameter in the diffusion equation. If we relax the isotropic diffusion condition and use anisotropic diffusion we are able to create a broader range of patterns. Assuming anisotropy, the reaction-diffusion equation can be generalized as:

$$
\frac{\mathscr{I} u}{\mathbb{I} t}=H(u, v)+\left(\nabla \cdot s_{u} \nabla\right) u,
$$

where $s_{u}$ is a symmetric positive definite diffusion tensor into which we will encode the vector field of interest. 
Assume that we are working on a finite domain $\left[a_{1}, b_{1}\right] \times$ $\left[a_{2}, b_{2}\right] \in \mathscr{R}^{2}$, and that we are given a regularly-spaced computational grid of size $N_{x} \times N_{y}$. At each point $\left(x_{i}, y_{j}\right), i=1, \ldots, N_{x}, j=$ $1, \ldots, N_{y}$ suppose that we are given a vector $\vec{v}_{i j}=\left(u_{i j}, v_{i j}\right)^{T}$. To embed this vector field into a tensor field, define $q_{i j}=\arctan \frac{v_{i j}}{u_{i j}} \in$ $[0,2 p]$.

We can now define a rotation matrix and its inverse based upon the angle determined above:

$$
\begin{gathered}
\mathbf{R}_{i j}=\left(\begin{array}{cc}
\cos q_{i j} & \sin q_{i j} \\
-\sin q_{i j} & \cos q_{i j}
\end{array}\right) \\
\mathbf{R}_{i j}^{-1}=\mathbf{R}_{i j}^{T}=\left(\begin{array}{cc}
\cos q_{i j} & -\sin q_{i j} \\
\sin q_{i j} & \cos q_{i j}
\end{array}\right)
\end{gathered}
$$

We now define a principal diffusivity matrix $\Lambda$ which is a diagonal matrix and gives the diffusivity coefficients along the two principal axes of diffusion:

$$
\Lambda_{i j}=\left(\begin{array}{cc}
\left(I_{1}\right)_{i j} & 0 \\
0 & \left(I_{2}\right)_{i j}
\end{array}\right)
$$

where $\left(I_{1}\right)_{i j}$ is the diffusivity in the first principal direction and $\left(I_{2}\right)_{i j}$ is the diffusivity in the second principal direction.

With the definitions above we can define a diffusivity tensor $s_{\mathbf{i j}}$ based on our vector field as

$$
s_{\mathbf{i j}}=\mathbf{R}_{i j}^{T} \Lambda_{i j} \mathbf{R}_{i j}
$$

We then combine the spatially nonuniform anisotropic diffusion matrix with a discrete finite difference Laplacian as outlined in [9] in a manner that maintains second-order convergence. With this control, we now have our desired mapping between the vector orientation and the diffusion kinetics. Witkin and Kass [32] took a similar approach for creating 2D texture patterns but assumed a spatially uniform anisotropic diffusion matrix.

\subsection{Directional Texturing}

The final step in the mapping process is to encode directional information in the pattern created. Up to this point we have described the use of a reaction-diffusion model for generating non-specific patterns. The most common pattern formed using a reaction-diffusion model is a spot pattern. The exact formation of the spot pattern will be discussed in the following section. Assuming an oriented elliptical spot pattern, we show the direction by texturing the spots with a contrasting light to dark fading texture. The texturing is local for each spot using the following steps:

Step 1: Assuming dark spots on a light background, normalize the image values to be between 0 and 255 .

Step 2: Find the centroid of each spot by first thresholding to remove all pixels with a value greater than 64 . Second, thin the remaining pixels to into single pixels [21]. Label the remaining single pixels as the centroid.

Step 3: For each centroid find all connected pixels with a value less than 128.

Step 4: For each connected pixel, calculate the dot product between the vector form by the centroid and the connected pixel and the underlying vector field value at the centroid.

Step 5: Normalize the dot product to be between 0 and 1 based on the minimum and maximum dot product values within the spot.

Step 6. If the normalized dot product is less than .9, interpolate between the minimum and maximum gray scales in the spot. This becomes the new gray scale value that gives the dark to light fading on the spot. Otherwise the gray scale is set to be 255 , which produces a contrasting light tip on the spot.
The texturing does not change the size or orientation of the spots; merely highlights the direction using values that maintain their natural appearance in the image. A light to dark fading provides directional cues because the fading has a natural strong to weak association. Wegenkittl et al. [30], took a similar approach to create oriented streamlines. The texturing is fully demonstrated in the following section.

\section{IMAGE FORMATION}

A reaction-diffusion image is created using a forward Euler integration on the discrete version of Equation (5) for $a$ and $b$ until a dynamic equilibrium state is reached, at which time, a stable pattern will have formed. We have found that using a cell size of 1.0 and a step size of 0.5 provides a balance between numerical stability and the pattern formation.

Figure (1a) shows a spot pattern created with the Turing model. Analysis of the image shows that the spot placement is balanced. That is, there is a uniform density of spots with equal spacing around them. This balancing process can be observed during the integration process when a spot begins to form in an area of lower concentration. Other nearby spots adjust themselves so they are not too close to the newly formed spot. Sometimes this adjustment may come in the form of a change in the position of the spots or when one or more of the spots disappears and its concentration is absorbed by remaining spots. This natural organization is one of the properties of reaction-diffusion equations that makes them very useful for visualization purposes.

Circular spots alone do not show magnitude, orientation, or direction. As discussed previously, to show magnitude we scale the spots using the reaction rate, $s$ to reflect the vector magnitude, Figure (1b). To show orientation we compress the spots into elliptical shapes by applying an anisotropic diffusion matrix where the values along the principal axis have a 3:1 ratio. Next, we rotate the diffusion matrix for each vector so that the ellipse's major axis is aligned with the vector field. Once the system comes to a dynamic equilibrium and the spots have formed, the light to dark texture is applied to each spot to show direction, Figure (2a). Figure (2b) shows the magnitude, orientation, and direction combined.

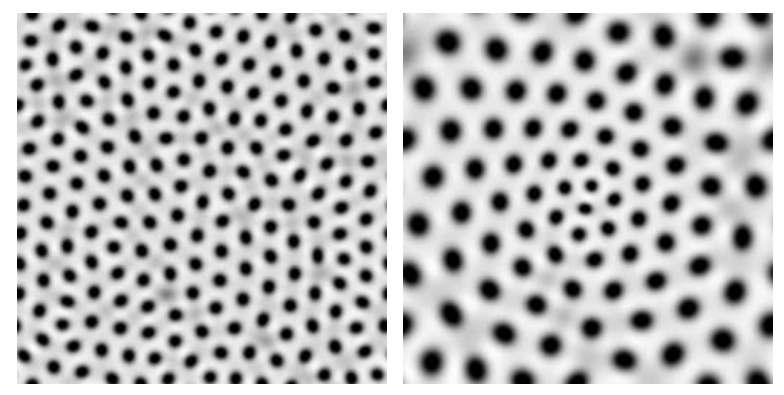

Figure 1: (a) Reaction-Diffusion visualization of circular spots of constant size and (b) variable size.

We now show the application of our reaction-diffusion model to visualize a set of idealized vector fields. Our goal was to see if it was possible to capture the nature of different types of vector fields. These fields include the electric field from a dipole and an electrostatic charge, Figure (3); and a vector field for a saddle and a sink, Figure (4). In each vector field the magnitude and orientation changes smoothly. The properties of vector field can easily be discerned as the ellipsoidal spots have the correct size and orientation. In the case of the circular, saddle, and sink vector fields, a change in the vector magnitude occurs as the vector field moves away from the center and is shown by a corresponding change in the spot size. However, in Figures (2-4), the images have several spots that did 


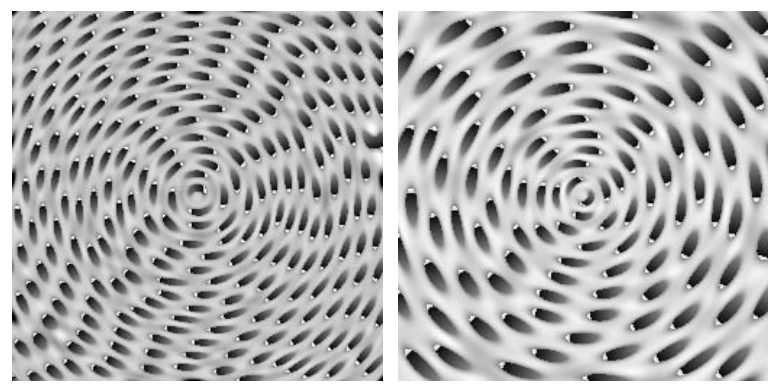

Figure 2: (a) Reaction-Diffusion visualization of a circular vector field showing orientation and direction and (b) magnitude, orientation, and direction.

not form very well, appearing to be smeared together. This is attributable to the variance of the formation and degradation factors, $a$ and $b$ which need to have enough variance to perturb the system but not so much as to cause irregularities in the patterns.

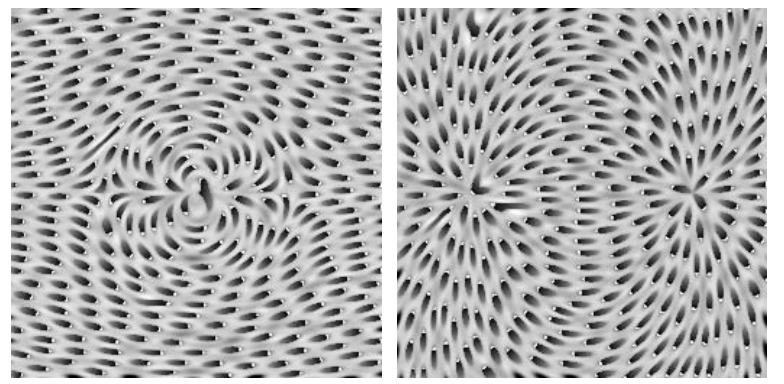

Figure 3: (a) Reaction-Diffusion visualization the electric field from a dipole and (b) an electrostatic charge.

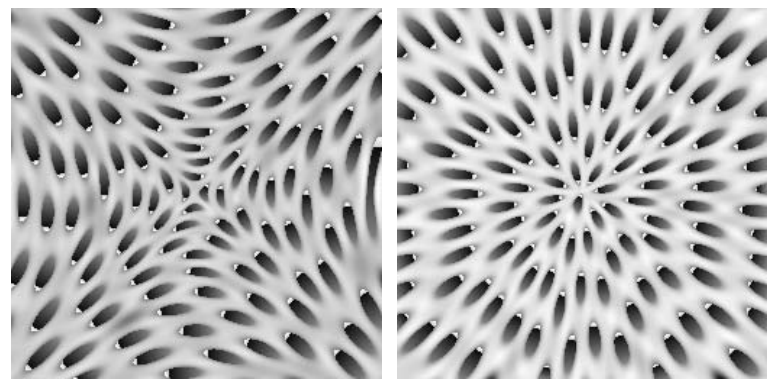

Figure 4: (a) Reaction-Diffusion visualization of a saddle vector field and (b) a sink vector field.

One of the unique features of using a reaction-diffusion model with anisotropic diffusion as we have done is that the spot, although appearing random, naturally align themselves into pseudo streamlines. Further, when the vector field is curved, the spots are not perfectly elliptical, but rather a bean-like shape. This is due to the spatially nonuniform anisotropy influencing the overall spot shape. Another feature of the reaction-diffusion model is that, due to the diffusion, faint streaks emanate from the ends of the spots. These streaks act to connect the spots, further aiding in visualizing the vector field.

This aligning, bending, and streaking all give the observer cues to the underlying vector field. But critical areas may also be of interest. This is another area where the reaction-diffusion model gives visual cues. For instance, at locations where the vector field is diverging, the spots are no longer elliptical but assume odd shapes. If the vector field is diverging equally in all directions the spots will be circular. As such, oddly shaped or circular spots could indicate, critical areas, or as will be demonstrated in the next section, the location of a large uncertainty in orientation. These are locations the observer may want to inspect further. For instance, in Figures (2-4) the spots are elliptical and are aligned with the vector field throughout the image except at the critical areas.

We have shown that it is possible to view different vector fields using a reaction-diffusion model. However, when implementing the reaction-diffusion method, a question regarding resolution arises: what is the minimum resolution required for individual features to be seen? By its nature, the process of diffusion acts to smooth, lowering high concentrations and raising low concentrations. As such, it is possible to lose individual features that are significantly different than their neighbors.

To determine the minimum resolution at which features can be seen, we oversampled a vector field until it was possible to see the impact of a single vector that was significantly different in both magnitude and orientation than its surrounding, otherwise constant neighbors. This is demonstrated in Figures (5) and (6) for both magnitude and orientation, respectively. It is not until there is an oversampling of eight times the original sample that the magnitude will significantly impact its neighbors to be visually noticeable. Similarly, it takes an oversampling of eight times for the angle to impact its neighbors. Unfortunately, for large vector fields, oversampling is not always practical because it may require significantly more computational time. As such, when visualizing a vector field without oversampling features less than eight nodes in size may be smoothed out.

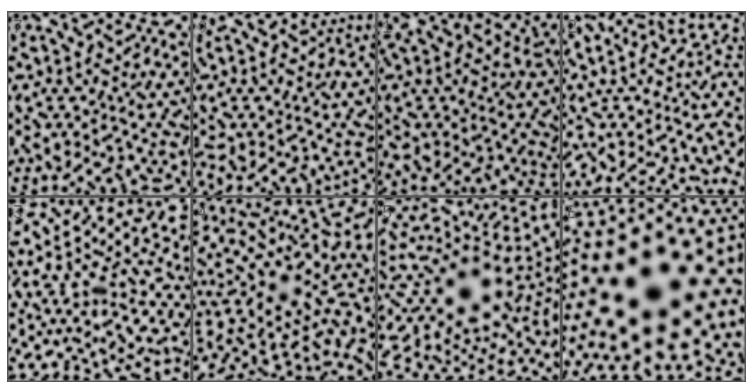

Figure 5: Effect of a single value on the spot size with an oversampling of $0,1,2,4,8,16,32$, and 64 times.

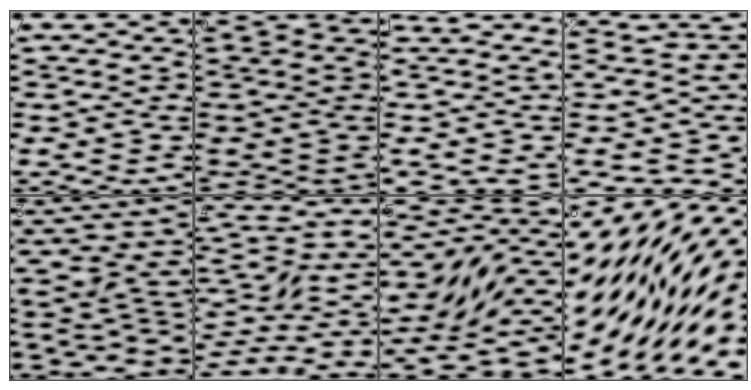

Figure 6: Effect of a single value on the spot orientation with an oversampling of $0,1,2,4,8,16,32$, and 64 times.

\subsection{Uncertainty Measurements}

In the previous examples, we have fixed the amount of anisotropy in the diffusion matrix. However, this is not necessary. If we allow the anisotropy to vary, we can map and visually represent another variable. In this case, we define the amount of anisotropy to be the ratio of the values along the principal axes in the diffusion matrix. When the amount of anisotropy is small, the spot formed is circular. Where when the anisotropy is high, the spot formed is elliptical, at 
times, almost to the point of being a thick line. This difference is very well suited to mapping an orientation uncertainty. When the orientation uncertainty is very small the spot maintains its elliptical shape, reflecting a precise orientation. When the uncertainty is very high, the spot is more circular, reflecting the uncertainty in the orientation. In the previous examples, the ratio of the values along the principal axes in the diffusion tensor was fixed at 3:1. We now allow it to vary between 5:3 and 7:1. This is demonstrated in Figure (7) where the uncertainty is a function of the angular position. In a subsequent example, instead of encoding the uncertainty, we encode the vorticity of the vector field,

$$
w_{i j}=\left(\nabla \times u_{i j}\right)
$$

which can be visualized as a scalar in $2 \mathrm{D}$.

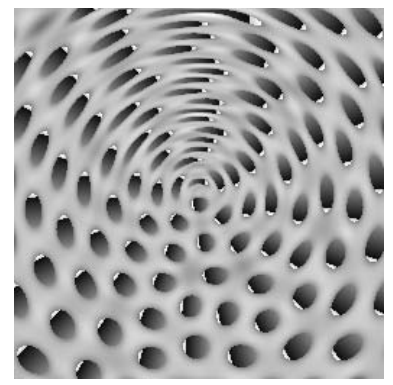

Figure 7: Reaction-Diffusion visualization of orientation uncertainty. The orientation uncertainty is a function of the angular position.

\subsection{Augmentation and Automatic Streamline/Glyph Place- ment}

Figure (8) shows our reaction-diffusion model for visualizing an idealized vector field that contains three saddles and two vortexes. To augment the LIC image we have taken Kirby et al.'s [12] painter's approach by using a LIC image as an undercoat with the reaction-diffusion as the topcoat. In addition to the trending pattern formed by the spots, the brush stroke appearance from the LIC image enhances the ability of the observer to follow the vector field, while the spots provide magnitude and direction.

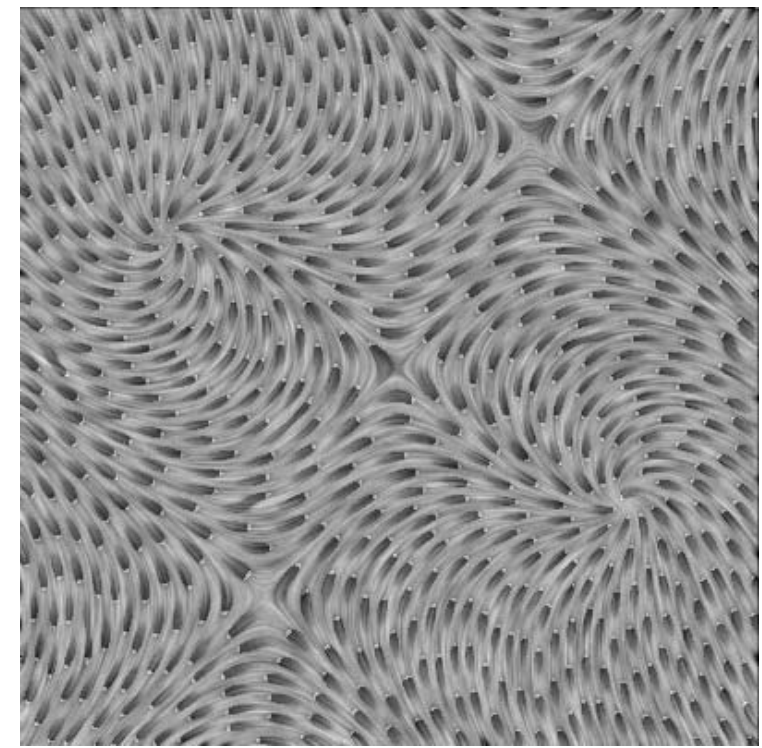

Figure 8: Reaction-Diffusion visualization of multiple vector fields using an underlying LIC image as a base coat.
One of the features of using a reaction-diffusion model is that it also provides a mechanism for automatic streamline or glyph placement. By finding the centroid of each spot using standard image processing techniques [21], it can serve as a seed point for placing a streamline, Figure (9) or a glyph, Figure (10). Although there is one streamline per spot in Figure (9), there appears to be fewer streamlines because of the their alignment and slight overlap. Because the density of the spots is based on the magnitude of the underlying vector field in Figure (10) it was possible to scale the arrow glyphs to reflect the vector magnitude without causing occlusion. Further, because of the density relationship, the number of glyphs also reflects, albeit inversely, the magnitude of the vector field. If a uniform density is desired then all that needs to be done is to run the reaction-diffusion using a constant magnitude term which would then determine the density.

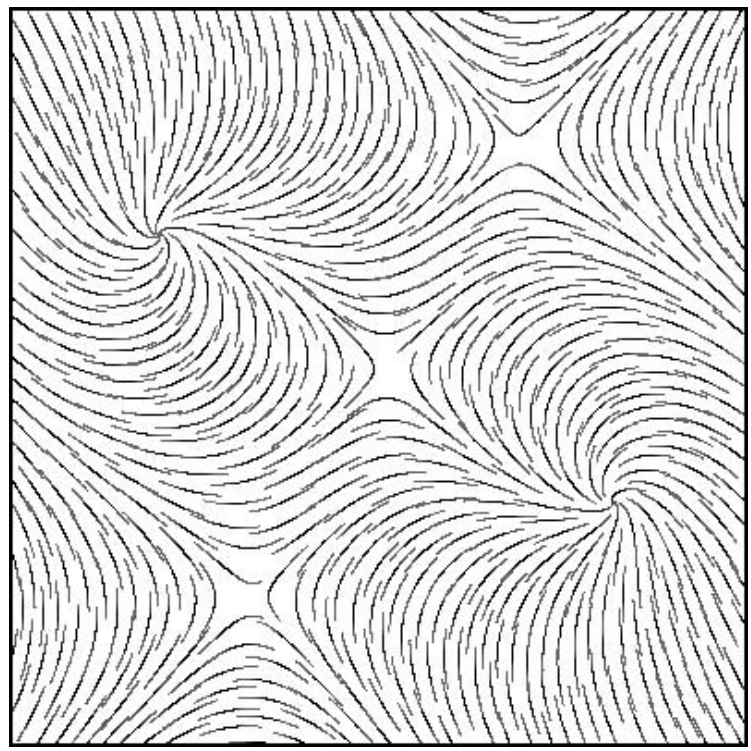

Figure 9: Automatic streamline placement based on the spot centroids in Figure (8).

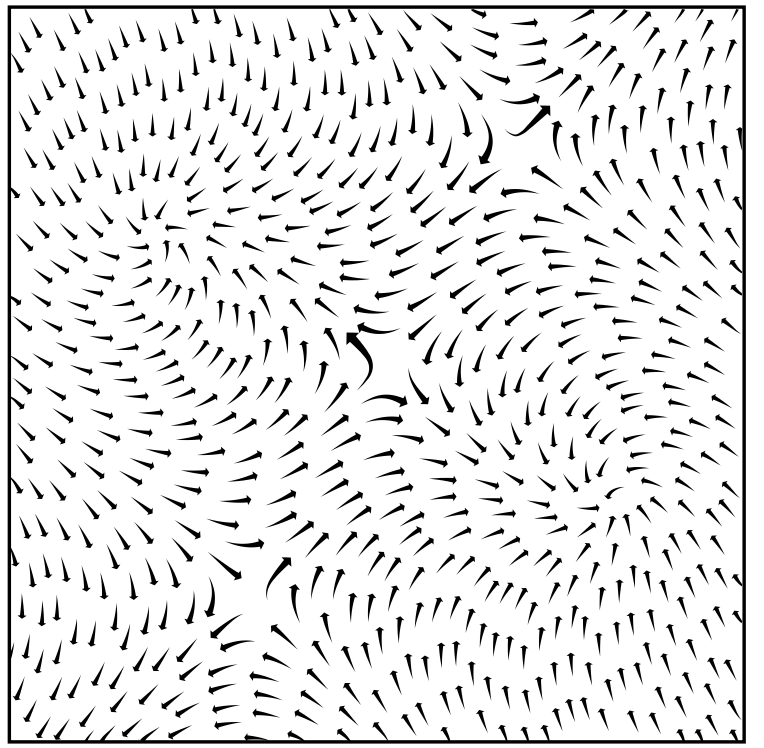

Figure 10: Automatic arrow glyphs placement based on the spot centroids in Figure (8). 


\section{Results ANd Discussion}

We now apply our reaction-diffusion model to a numerical simulation of the nonlinear magneto-hydrodynamics (MHD) that occur in the DIII-D tokamak nuclear fusion reactor. The vector field shown in Figures (11-13) is a reoriented two-dimensional slice of the magnetic field in the Tokamak reactor. In Figure (11), just the magnitude and vorticity of the vector field is visualized with no orientation information. The greater the vorticity, the more symmetric the spots become. This gives a good example of how this technique can be used for visualizing two scalar values. Figure (12) is the same vector field showing the orientation and direction. Finally, in Figure (13), the vector field is shown with magnitude, orientation, and direction. In Figure (14), we have encoded the magnitude, orientation, direction, and vorticity of the vector field. Although the spots in Figure (14) appear in different locations than in Figure (13) a comparison of the two images shows a significant difference in the spot shape in those areas with high vorticity.

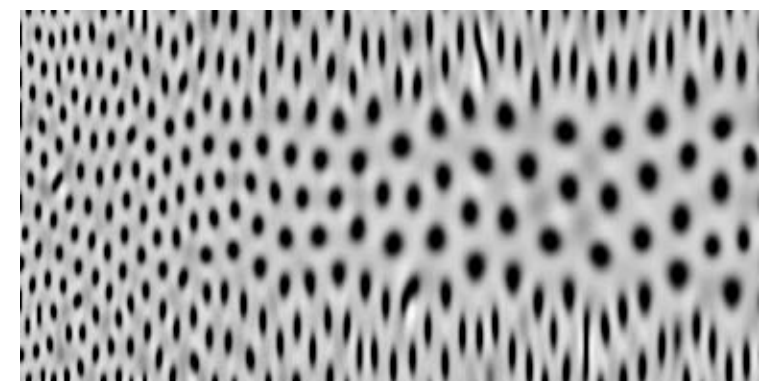

Figure 11: Reaction-Diffusion visualization of a MHD Magnetic vector field. Magnitude and Vorticity are shown.

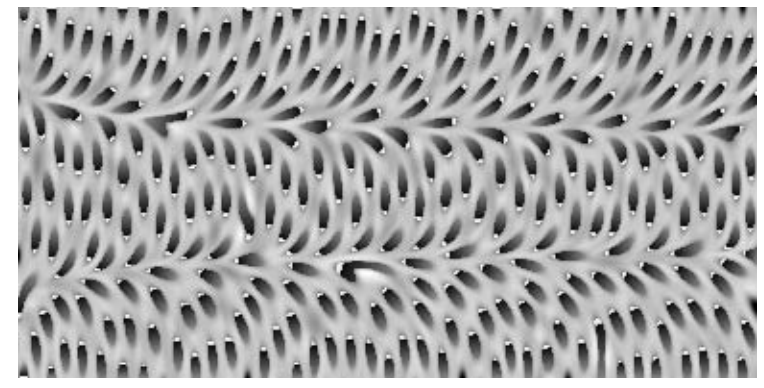

Figure 12: Reaction-Diffusion visualization of a MHD Magnetic vector field. Orientation and direction are shown.

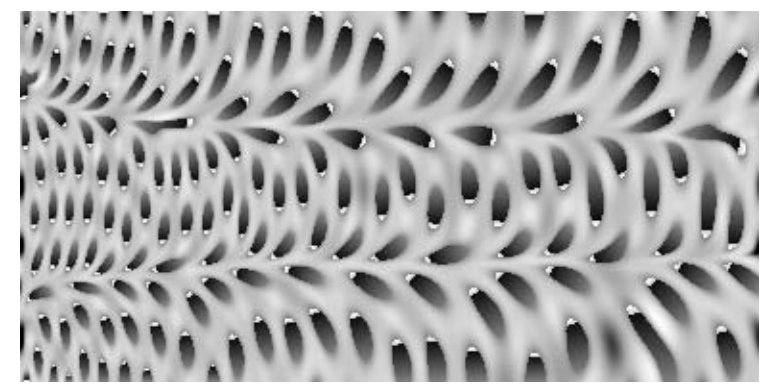

Figure 13: Reaction-Diffusion visualization of a MHD Magnetic vector field. Magnitude, orientation, and direction are shown.

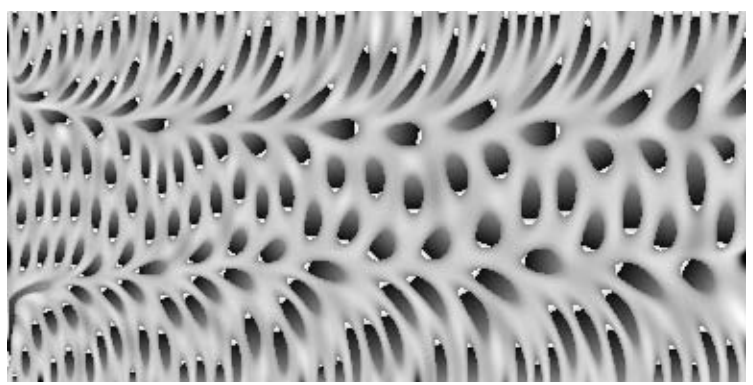

Figure 14: Reaction-Diffusion visualization of a MHD Magnetic vector field. Magnitude, orientation, direction, and vorticity are shown.

\subsection{Relationship to Other Vector Field Visualization Tech- niques}

It is also possible to set the reaction rate to zero and use just the diffusion tensor to create other types of visualizations. To produce a LIC-like image, we change the diffusion tensor to be 1D, Figure (15a). To produce convective patterns, such as those proposed by Preußer [20], we use a highly anisotropic 2D diffusion tensor, Figure (15b). This shows that the diffusion model used is consistent with previously published results.
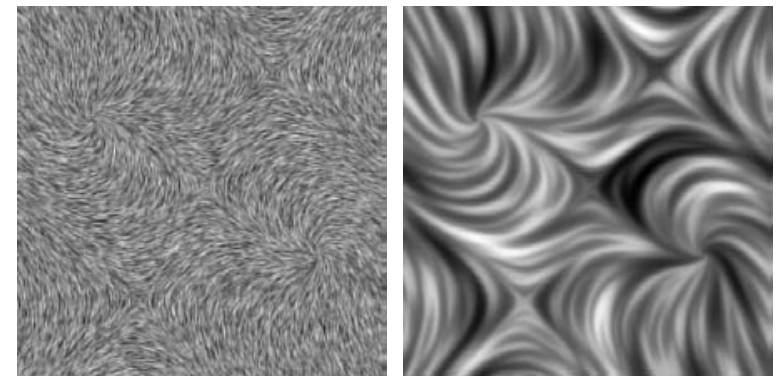

Figure 15: (a) Visualization with a LIC like appearance and (b) with a convective patterns using just the diffusion kinetics.

\subsection{Comparison with Other Vector Field Visualization Tech- niques}

We now compare the reaction-diffusion images with four different vector field visualization techniques. Figure (16) shows vector glyphs at regular intervals [25], Figure (17) shows oriented ellipses based on a Poisson distribution [12], Figure (18) shows a line integral convolution [3], and Figure (19) shows image guided streamlines [28]

Placing glyphs at regular intervals is much simpler and quicker than using a reaction-diffusion model, but, as previously discussed, occlusion can be a problem. Using a random Poisson distribution solves the occlusion problem but fails to provide any organization, which is often a key to producing an effective visualization. Using a reaction-diffusion model overcomes the occlusion problem because the spots have a density that is based upon the vector magnitude. Another problem with regular and random intervals is that they may mislead the eye by forming a pattern that may not be part of actual vector field. Conversely, the reaction-diffusion model forms spots in a pattern that follows the underlying structure of the field.

When comparing the reaction-diffusion method to LIC, we can see that both techniques visualize the vector field in a manner that is natural and easy to observe by producing a dense image representation of the vector field field. With reaction-diffusion images, different reaction rates produce spots at different densities. The less dense the spots, the greater the chance that areas of interest may 
be missed. However, images with a high density of spots may be difficult to view because of the Moray patterns that can form. As such, the density of the spots is a critical component for an effective reaction-diffusion vector field visualization. Currently, the only way to control the density is by using different reaction rates. However, we are also investigating different reaction-diffusion models, such as the Gray-Scott model.

Unlike traditional LIC images, which do not contain magnitude or direction information, the reaction-diffusion model is able to naturally incorporate this information into the visualization. Including the magnitude and direction greatly enhances the visualization. LIC images, along with other noise-based techniques, can be extended to show the magnitude, but these techniques do so at a loss of vector field detail because of blurring used to emphasize the magnitude $[5,13]$.

Next, we compare the reaction-diffusion image to a visualization using the image-guided streamline technique developed by Turk and Banks [28]. Both techniques are similar in that both are able to show magnitude, orientation, and direction. However, the reactiondiffusion technique represents magnitude more intuitively than the image guided technique. This is because instead of using length to represent magnitude the reaction-diffusion technique uses a width, which is more intuitive.

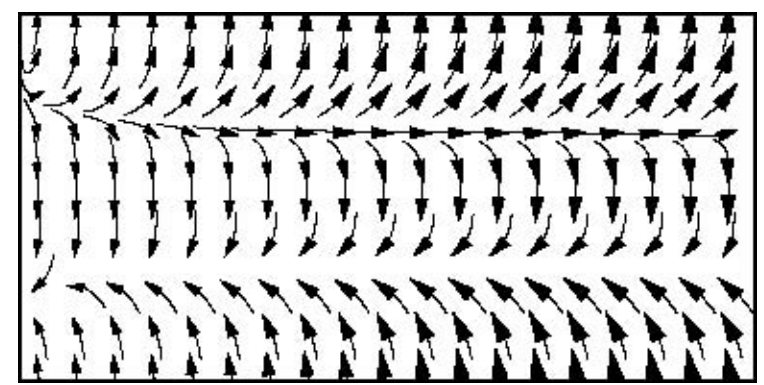

Figure 16: Uniform sampled vector glyph image of the vector field used in Figure (11).

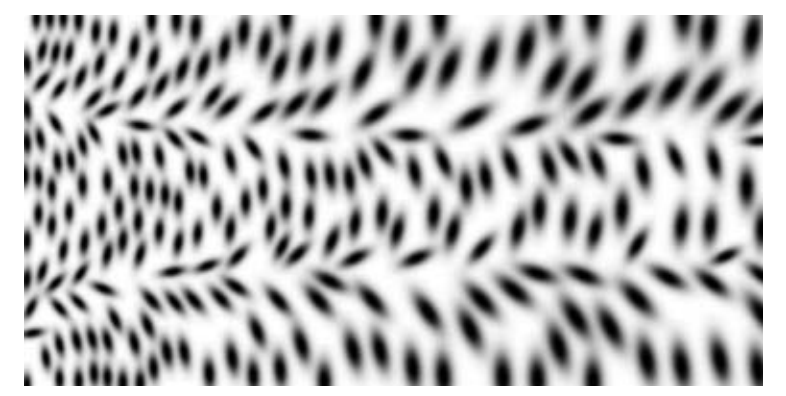

Figure 17: Randomly placed ellipse image of the vector field used in Figure (11).

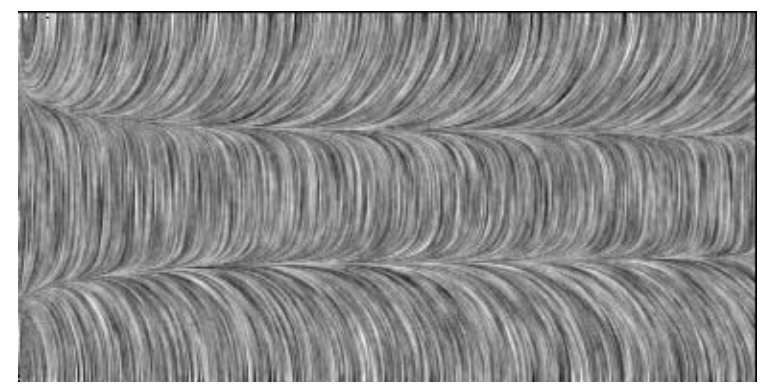

Figure 18: LIC image of the vector field used in Figure (11).

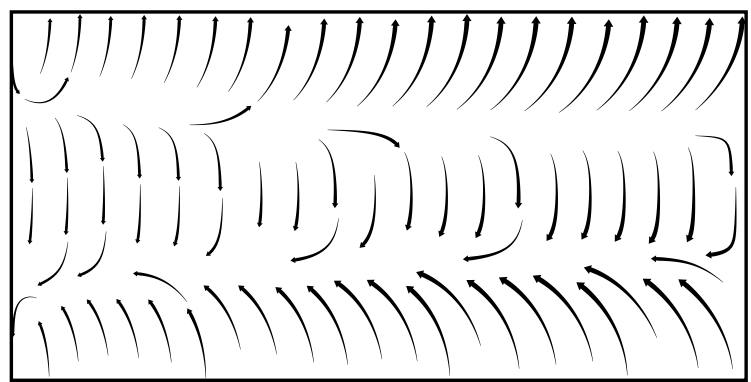

Figure 19: Image guided streamlines generated by Turk and Bank's algorithm of the vector field used in Figure (11).

One of the drawbacks of using a reaction-diffusion model compared to the other techniques is the computational expense. Using an explicit formulation, the patterns take $15-25 \mathrm{k}$ iterations to form and become stable. Using a GPU implementation based on Lefohn et al. [15] this takes less than a minute. Whereas it is possible to produce LIC images at interactive rates [2]. We are currently investigating GPU based multi-grid and implicit integration techniques, which should reduce the computational expense.

An additional problem that can occur during pattern formation is that spots can form and fail to separate, as shown in Figure (3a). Where this happens is random and appears to be dependent on the initial conditions. We have observed that it tends to happen more frequently with smaller spots.

One of the greatest benefits of using a reaction-diffusion model is the ability to seamlessly integrate uncertainty measurements in the model. None of the other techniques, with the exception of vector glyphs, are able to show uncertainty as part of their representation [17]. Although vector glyphs can show uncertainty, occlusion remains a problem in their use.

An additional benefit is that the reaction-diffusion technique can be used alone or to augment other techniques. Furthermore, it is possible to use the centroids of the spots to provide a set of seed points for placing streamlines and scaled glyphs.

\section{CONClusions AND Future Work}

We have introduced the use of a reaction-diffusion model that can produce patterns with different shapes, sizes, orientations, and directions for visualizing vector fields. We are able to control the pattern formation by mapping two of the vector field components, orientation and magnitude, to the diffusion and reaction kinetics, respectively. In addition, we also can map an orientation uncertainty to the diffusion kinetics. This mapping produces a spot pattern that is highly representative of the underlying vector field. To show direction we have applied a light to dark fading texture to each spot.

The principle advantage of the reaction-diffusion model over existing vector field visualization techniques is that the pattern size and density that naturally arises from the reaction-diffusion model accurately represents the underlying vector field. Further, the shape of the pattern (e.g. the spots) not only contains information concerning magnitude, orientation, and direction but also may contain other information, such as uncertainty or vorticity.

We have also demonstrated the use of the reaction-diffusion model for the automatic placement of streamlines or glyphs and shown how it can be augment other techniques. Although we have not used color to highlight certain features, one could easily incorporate color to further enhance the visual attributes.

Future work includes extension of the reaction-diffusion algorithm to three dimensions. In such an extension, the reaction kinetics remain the same; only the diffusion kinetics must be extended. The output is a three-dimensional texture that can be volume ren- 
dered using various techniques or may be applied to two dimensional surfaces. The image generated would have similar characteristics to those generated by Kindlmann and Weinstein [11] and Chambers and Rockwood [4] and unfortunately suffer from the same visualization problems.

Finally, there are a number of perceptual issues that require further investigation, including a formal user study such as the one performed by Laidlaw et. al. [14] to determine the effectiveness of the reaction-diffusion visualization technique in comparison to other vector field visualization techniques. One area of particular interest is quantifying the effectiveness of the natural patterns that form from using a reaction-diffusion model.

\section{ACKNOWLEDgments}

This work was supported, in part, by the DOE SciDAC National Fusion Collaboratory and by grants from NSF, NIH, and DOE. The authors are grateful to Miriah Meyer for generating the images using a GPU implementation, Ravi Samtaney and Scott Kruger for providing data and to Greg Turk for providing the code for the image guided streamline.

\section{APPENDiX}

One of the difficulties in using a reaction-diffusion model is the inherent instability of the system. Below are the parameters used to obtain the stable pattern shown in Figure (1a), which are applied to Eqs. (1-4) using a discrete central difference Laplacian on a uniform grid.

$a=4.0$

$b=4.0$

$D_{a}=1.0 / 4.0$

$D_{b}=1.0 / 16.0$

$a=16.0 \pm 1 \%$

$b=12.0 \pm 1 \%$

$s=1.0 / 64.0$

\section{REFERENCES}

[1] D.C. Banks and B.A. Singer. A predictor-corrector technique for visualizing steady flow. IEEE Transactions on Visualization and Computer Graphics, 2:151-163, 1995.

[2] U. Bordoloi and H.W. Shen. Hardware accelerated interactive vector field visualization: A level of detail approach. Computer Graphics Forum, 21(3), 2002.

[3] B. Cabral and C. Leedom. Imaging vector fields using line integral convolution. In Proceedings of SIGGRAPH 93, pages 263-270. ACM SIGGRAPH, 1993.

[4] P. Chambers and A. Rockwood. Visualization of solid reactiondiffusion systems. IEEE Computer Graphics and Applications, pages 7-11, 1995.

[5] W.C. de Leeuw and J.J. van Wijk. Enhanced spot noise for vector field visualization. In IEEE Visualization 95 Proceedings, pages 233-239, 1995.

[6] H. Garcke, T. Preußer, M. Rumpf, A. Telea, U. Weikard, and J. van Wijk. A phase field model for continuous clustering on vectot fields. IEEE Transaction on Visualization and Computer Graphics, 7(3):230-241, 2001.

[7] J. Helman and L. Hesselink. Visualizing vector field topology in fluid flows. IEEE Computer Graphics and Applications, May 1991.

[8] L. Hultquist. Constructing stream surfaces in steady 3D vector fields. In Proceedings of IEEE Visualization '92, pages 171-177. IEEE Computer Society Press, 1992.

[9] G.E. Karniadakis and R. M. Kirby II. Parallel Scientific Computing in $C++$ and MPI. Cambridge, New York, 2003.
[10] D.N. Kenwright and G.D. Mallinson. A 3-D streamline tracking algorithm using dual stream functions. In IEEE Visualization 92, pages 62-68, 1992.

[11] G.L. Kindlmann, D.M. Weinstein, and D. Hart. Strategies for direct volume rendering of diffusion tensor fields. IEEE Trans. Visualization and Computer Graphics, 6(2):124-138, 2000.

[12] R.M. Kirby, H. Marmanis, and D.H. Laidlaw. Visualizing multivalued data from $2 \mathrm{~d}$ incompressible flows using concepts from painting. In Proceedings of the IEEE Visualization 99, 1999.

[13] Ming-Hoe Kiu and David C. Banks. Multi-frequency noise for LIC. In Roni Yagel and Gregory M. Nielson, editors, IEEE Visualization '96, pages 121-126, 1996

[14] David H. Laidlaw, Robert M. Kirby, Cullen D. Jackson, J. Scott Davidson, Timothy S. Miller, Marco da Silva, William H. Warren, and Michael Tarr. Comparing $2 \mathrm{~d}$ vector field visualization methods: A user study. IEEE Transactions on Visualization and Computer Graphics, Accepted for Publication, 2004.

[15] A.E. Lefohn, J.M. Kniss, C.D. Hansen, and R. Whitaker. Interactive deformation and visualization of level set surfaces using graphics hardware. In IEEE Visualization 2003, pages 75-82, October 2003.

[16] J.D. Murray. Mathematical Biology. Springer-Verlag, New York, 1989.

[17] A.T. Pang, C.M. Wittenbrink, and S.K. Lodha. Approaches to uncertainty visualization. The Visual Computer, 13(8):370-390, November 1997.

[18] F. Post, B. Vrolijk, H. Hauser, R.S. Laramee, and H. Doleisch. Feature extraction and visualization of flow fields. Eurographics 2002, pages 69-100, 2002.

[19] F.J. Post, T. van Walsum, Post F.H., and D. Silver. Iconic techniques for feature visualization. In IEEE Visualization 95, pages 288-295, 1995.

[20] T. Preußer and M. Rumpf. Anisotropic nonlinear diffusion in flow visualization. In Proceedings of Visualization '99, pages 325-332. IEEE Computer Society Press, Los Alamitos, CA, October 1999.

[21] A. Rosenfeld and A.Kak. Digital Picture Processing. Academic Press, 1982.

[22] C. Schroeder, W. Volpe and W. Lorensen. The stream polygon: A technique for 3D vector field visualization. In IEEE Visualization '91, pages 126-132. IEEE Press, 1991.

[23] H.W. Shen, C.R. Johnson, and K.L. Ma. Global and local vector field visualization using enhanced line integral convolution. In Symposium on Volume Visualization, pages 63-70. IEEE Press, 1996.

[24] A. Telea and J. J. van Wijk. Simplified representation of vector fields. In Proceedings of the IEEE Visualization 99, pages 35-42, 1999.

[25] E.R. Tufte. The Visual Display of Quantitative Information. Graphics Press, Cheshire, Conn., 2nd edition, 2001.

[26] A.M. Turing. The chemical basis of morphogenesis. Phil. Trans. Roy. Soc. Lond., B237:37-72, 1952.

[27] G. Turk. Generating textures on arbitrary surfaces using reactiondiffusion. Computer Graphics (SIGGRAPH), 25(4):289-298, 1991

[28] G. Turk and D. Banks. Image-guided streamline placement. Computer Graphics (SIGGRAPH), pages 453-460, 1996.

[29] J.J. van Wijk. Spot noise: Texture synthesis for data visualization. Computer Graphics, 25(4):309-318, 1991.

[30] R. Wegenkittl and Eduard Groller. Fast oriented line convolution for vector field visualization via the internet. In Visualization '97, pages 309-316, Phoenix, AZ., 1997. IEEE Press.

[31] R. Westermann, C. Johnson, and T. Ertl. A level-set method for flow visualization. In IEEE Visualization 2000, pages 147-154, Salt Lake City, 2000. IEEE Computer Society.

[32] A. Witkin and Mi. Kass. Reaction-diffusion textures. Computer Graphics (SIGGRAPH), 25(4):299-308, 1991.

[33] M. Zockler, D. Stalling, and H-C. Hege. Interactive visualization of 3D-vector FIelds using illuminated stream lines. In Visualization '96, San Francisco, CA., 1996. IEEE Press. 